\title{
Perspective Randomized-controlled clinical trial in patients submitted to Self-Expanding Metal Stents for Colo-rectal cancer with bowel obstruction. Single center experience. Preliminary data
}

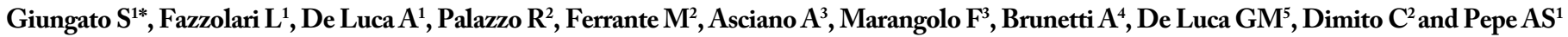

${ }^{1}$ Unit of Surgery and Endoscopy, Department of Emergency Surgery, "San Pio" Castellaneta Hospital, Taranto, Italy

${ }^{2}$ Unit of Anesthesia, Department of Emergency, "San Pio" Castellaneta Hospital, Taranto, Italy

${ }^{3}$ Unit of Radiology, Department of Emergency, "San Pio" Castellaneta Hospital, Taranto, Italy

${ }^{4}$ Unit of Oncology, Department of Medicine, "San Pio" Castellaneta Hospital, Taranto, Italy

${ }^{5}$ Unit of General Surgery "V. Bonomo”, Department of Emergency Surgery, University of Medical School of Bari Policlinico, Italy

\begin{abstract}
Introduction: Since the early 1990s, self- expanding metal stents (SEMS) have been used to treat malignant colonic obstruction. This kind of endoscopic procedure is mostly used for palliative treatment of colo-rectal cancer buti it is also used as "bridge to surgery".

Materials and methods: This perspective randomized-controlled clinical trial was performed from January 2017 to October 2019. We have recruited all patients admitted in our Emergency Hospital with diagnosis of colonic obstruction. All patients submitted to CT-scan. Patients was divided in 3 Groups: Group A (control group, composed by patients submitted only to emergency surgery) were 17; Group B (patients submitted to SEMS positioning and surgery) were 10; Group C (patients submitted to only SEMS positioning) were 14. The same Metal stent was used for all patient submitted to SEMS positioning (Colonic WallflexTM Boston Scientific $9 \mathrm{~cm}$ ).

Results: Analysis of comparison between Group A vs B highlighted statistical significance for diverting stoma performe (p-value: one-tailed 0.00035; two-tailed 0.00075 ; $\mathrm{p}<0.001$ ), equal $70,5 \%$ of patients (12 out of 17 ). Also the comparison between Group A and B, in relation of survival rate (13 patients out of 17 , equal to $70,58 \%$ in Group A and 10 patients out of 10, equal to 100\%, in Group B after two years follow-up), statistical significance shows (p-value one-tailed 0.0010; twotailed; p $<0.005$ ). Comparison of Group B plus C (24 patients), submitted to SEMS, shows time procedure was 25,17 minutes (range 10 - 40) and nobody patients showed stent dislocation or bowel obstruction signs after $48 \mathrm{~h}$ from endoscopic procedure. 1 case of Tenesmo in rectal SEMS and 2 colonic perforation after 73 days and 15 days (8\%). They submitted early food intake. In our clinical controlled trial, even if is a preliminary data, demonstred that SEMS positioning is a the best palliative procedure with high success rate $(95,8 \%)$ and a good survival. Mean days of hospitalization of 3,4 days.
\end{abstract}

Conclusion: Our clinical randomized trial shows that SEMS positioning is feasibility as palliative therapy for obstructive colo-rectal cancer and it's safe procedure as "bridge to surgery". Other patients are ruled but a greater follow-up will be necessary to make our study more effective.

\section{Introduction}

Endoscopic treatment of neoplastic stenosis with self-expanding metal stent (SEMS) positioning is increased in the last years but this procedure is not yet fully accepted. This procedure is mainly used for treatment of neoplastic obstruction of esophagus, duodenum and colon and mostly for palliative therapy. Although, most of the time, endoscopic procedure is a palliative treatment but it is also used as "bridge to surgery" for curative setting.

SEMS positioning is a difficult procedure and needs of precise directors but is a feasible procedure in all hospitals with dedicated team composed by expert endoscopists, dedicated anesthesiologists and nurses.

The target of SEMS is to decompress the bowel and to avoid emergency surgical operations so to stabilize patients for submitting them to elective surgical operations, adjuvant chemo and/or radio therapy, or for palliative therapy without submitted them to emergency operations.
This perspective randomized controlled clinical trial wants to explane the real feasibility of SEMS positioning procedures in patients with colo-rectal neoplastic obstruction.

\section{Materials and methods}

This study was performed at Castellaneta Hospital, department of emergency surgery and endoscopy, Taranto, Italy, from Febbruary 2017 to October 2019.

${ }^{\star}$ Correspondence to: Giungato S, Unit of Surgery and Endoscopy, Department of Emergency Surgery, "San Pio" Castellaneta Hospital, Taranto, Italy, E-mail: simone.giungato@libero.it

Key words: colon cancer, stent, colonic obstruction, self expanding metal stent, controlled trial

Received: November 04, 2019; Accepted: November 21, 2019; Published: November 25, 2019 
We have ruled all patients admitted in emergency with colonic obstructive syndrome. Inclusion criteria was: Admitted in emergency; CT-SCAN showing obstruction of colon with dilatation of upstream bowel; informed consent to surgical or endoscopic procedure.

We have divided patients into three groups: Group A, control group, patients submitted to urgent surgery without SEMS positioning; Group B, patients submitted to SEMS positioning and after operation; Group C, patients submitted to SEMS positioning without operation because the presence to CT-SCAN of lung and liver metastasys (stage IV disease), or bilobar liver metastasis only associated to old age. Preventive colonoscopy was performed to all patients submitted to SEMS positioning.

Metal stent was performed by the same endoscopist (Group B and C) and colo-rectal resection of Group B was performed by the same two surgeons, while patients of Group A, control group, were submitted to operation by different surgeons. SEMS.

Wallflex ${ }^{\mathrm{TM}}$ Boston Scientific $9 \mathrm{~cm}$ colonic metal stent was used as

Operational endoscopic procedures were perfomed in deep sedation. All patients received fentanyl $(25-50 \mu \mathrm{g})$ and iv midazolam (2-3 mg) routinely before Propofol TCI.

The initial effect site concentration (Ce) of Propofol TCI system was set at $2-3 \mu \mathrm{g} / \mathrm{ml}$ for colonoscopy. Ce of propofol TCI was further titrated using $0.5 \mu \mathrm{g} / \mathrm{ml}$ steps. During procedures a bolus of fentanyl $(25-50 \mu \mathrm{g})$ could be added as rescue therapy. All patients received oxygen $21 /$ min via nasal cannula. Procedures began when patients did not respond to eyelid stimulation. Monitoring included electrocardiography (II lead), heart rate, peripheral oxygen saturation, non invasive blood pressure (every $5 \mathrm{~min}$ ), capnography, according to recent guidelines (1).

No severe complications, such as persistent hypoxia needing tracheal intubation or severe hypotension (sistolic pressure $<90 \mathrm{mmHg}$ ) occured. Bradycardia episodes $(\mathrm{HR}<50$ beats $/ \mathrm{min})$ were treated with iv atropine bolus infusion. No procedures was interrupted. In two cases respiratory depression needed manual ventilation via facial mask.

After SEMS positioning all patients submitted to water diet after 24 hours and food intake after X-Ray of abdomen control at 48 hours later to check correct positioning of metal stent.

We have analyzed of Group A: day of hospitalization, operation time, patients submitted to direct anasthomosis, number of patients with derivating stoma (ileostomy or colostomy), survival rate from 6 months to 2 years follow-up.

We have analyzed of Group B: day of hospitalization, operation time, success rate of the procedures, patient submitted to direct anasthomosis or diverting stoma, time before surgery after SEMS positioning and survival rate.

We have analyzed of Group C: day of hospitalization, success rate of the procedures, operation time, rate of complications such as death, perforation or dislocation of prothesis (early complications), long-term complications such as perforation, dislocation or bad canalization. Procedures were performed by the same endoscopist and nurse and the same anesthesiology team. Follow-up of patients was performed from 6 months to 2 years.

\section{Criteria of randomization}

According to guidelines of CONSORT 2010 randomized criteria we composed study groups [1] (Flow Chart). We used really

\section{CONSORT 2010 FLOW-CHURT}

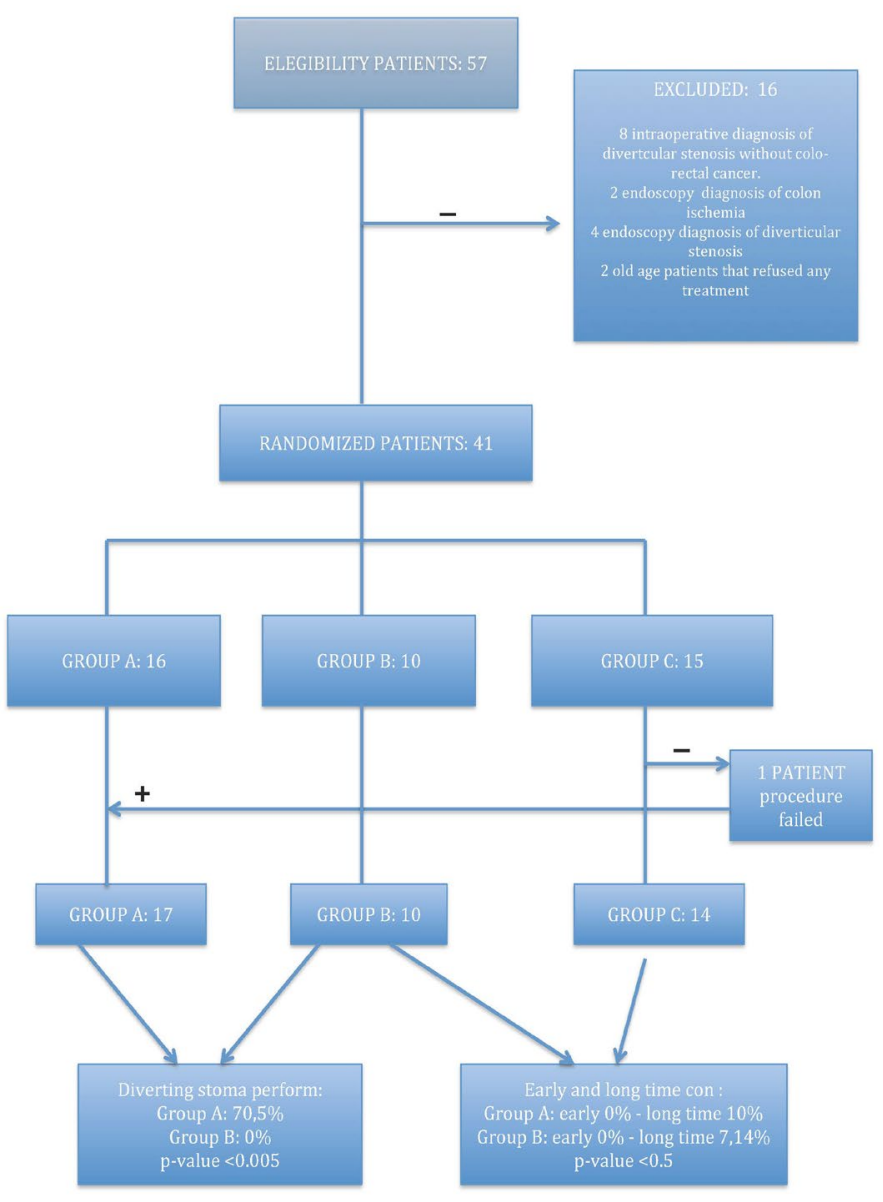

casual randomized criteria because pazients admitted in hospital with diagnosis of colonic obstruction was underwent operation, indipendently to other conditions, if the endoscopist was not present to performe SEMS positioning. This kind of randomization respects the clinical trials guidelines beacuse it allows a true casual randomization.

\section{Statistical analysis}

The comparison between two groups has been not perfomed using $x$ square because $x^{2}$ is calculated only used if all expected cell frequencies are equal to or greater than 5. For this reason was used Exact Fisher's Test for calculating p-value, with confidence interval of $95 \%$, giving a statistical significance for $\mathrm{p}$-value less then 0.05 .

$$
p=\frac{\left(\begin{array}{c}
A+C \\
A
\end{array}\right)\left(\begin{array}{c}
B+D \\
B
\end{array}\right)}{\left(\begin{array}{c}
N \\
A+B
\end{array}\right)}=\frac{(A+B) !(C+D) !(A+C) !(B+D) !}{A ! B ! C ! D ! N !}
$$

Exact Fisher's Test compared Group A and B for performing of diverting stoma (ileostomy or Colostomy); and survival rate of patients after two years follow-up. T-Student distribution was used for comparing operation time.

\section{Results}

From January 2017 to October 2019 we ruled 57 patients but of those, 16 patients were excluded: 8 because intraoperative and pathological 
Giungato S (2019) Perspective Randomized-controlled clinical trial in patients submitted to Self-Expanding Metal Stents for Colo-rectal cancer with bowel obstruction. Single center experience. Preliminary data

diagnosis was diverticular stenosis; 2 patients were excluded for preoperative endoscopical diagnosis of colon ischemia; 4 patients were excluded for pre operative diagnosis of diverticular stenosis and 2 old age patients were excluded because refused any treatment.

Of 41 patients remaining, 1 patients with endoscopical diagnosis of bowel obstruction for sigmoid cancer, is has been inserted in Control Group because SEMS positioning failed.

Patients have been divided in this way (Table 1): Group A, Control group, composed by 17 patients, with mean age 72,4 (range 62-83 years), mean days of hospitalization 5,9 days, operation time of 249,12 minutes and with survival rate of $88 \%$ at two years follow-up (15 patients out of 17), 2 patients dead for advanced disease and no response to postoperative chemotherapy. 12 patients out of 17 was submitted to colonic resection and diverting stoma.

Group B, SEMS plus Surgery, composed by 10 patients, with mean age of 71,9 years (range 58-83 years), operation time of 192,5 minutes with mean days of hospitalization equal to 14,4 days. All alive at two years follow-up and nobody submitted to intraoperative diverting stoma. Occlusive syndrome was resolved after SEMS positioning procedure in all patient.

Group C, SEMS without surgery, is composed by 14 patients, with mean age 86,6 years (range 78-92 years), and mean days of hospitalization of 3,4 days, with average survival equal to 7,2 month (range 15 days to 16 mesi), submitted, after hospital discharge, to chemotherapy.

Analysis of comparison between Group A vs B highlighted statistical significance for diverting stoma performe ( $p$-value: onetailed 0.00035; two-tailed 0.00075; $\mathrm{p}<0.001$ ), equal $70,5 \%$ of patients (12 out of 17). Also the comparison between Group A and B, in relation of survival rate (13 patients out of 17 , equal to $70,58 \%$, in Group A and 10 patients out of 10 , equal to $100 \%$, in Group B after two years followup, statistical significance shows ( $\mathrm{p}$-value one-tailed 0.0010; two-tailed; $\mathrm{p}<0.005$ ) (Table 2).

To evaluate the feasibility and safety of SEMS positioning, we analyzed Group B and C (Table 1), in according to time of procedure, state of bowel obstruction and perforated patients, at early time, during the procedure and long time, after at least 5 days to SEMS positioning. In both Groups, 24 patients, time procedure was 25,17 minutes (range 10-40) and nobody patients showed stent dislocation or bowel obstruction signs after $48 \mathrm{~h}$ from endoscopic procedure. 1 case of Tenesmo in rectal SEMS.

Table 1. Comparison among study Groups. Group A: Only emergency surgery; Group B: SEMS plus surgery; Group C: Only SEMS

\begin{tabular}{|c|c|c|c|}
\hline & Group A & Group b & Group c \\
\hline N. Patients & 17 & 10 & 14 \\
\hline Mean age & 72,4 (Range 62-83) & 71,9 (Range 58-83) & 86,6 (Range 78-92) \\
\hline $\begin{array}{l}\text { Mean days } \\
\text { hospitalization }\end{array}$ & 5,9 (Range $2-9$ ) $\pm 2,2$ & $\begin{array}{l}14,3 \text { (Range 10- } \\
25) \pm 5\end{array}$ & $3,4$ (Range $3-6) \pm 1$ \\
\hline $\begin{array}{l}\text { Mean survival } \\
(6 \mathrm{MONTH}-2 \mathrm{YR})\end{array}$ & $\begin{array}{l}88 \% \\
(15 / 17)\end{array}$ & $\begin{array}{l}100 \% \\
(10 / 10)\end{array}$ & $\begin{array}{l}50 \%-7,2 \text { months } \\
\text { (range } 15 \text { gg-16 } \\
\text { months) }\end{array}$ \\
\hline Diverting stoma & $70,58 \%(12 / 17)$ & $0 \%(0 / 10)$ & - \\
\hline
\end{tabular}

Table 2. Comparison Control Group vs Group B. Stasistically significance

\begin{tabular}{|c|c|c|c|}
\hline & GROUP A & GROUP B & P-value \\
\hline Operation time & $249,12 \pm 59,12$ & $192,5 \pm 72,7$ & $\mathrm{P}<0.025$ \\
\hline Survival rate & $88 \%(15 / 17)$ & $100 \%(10 / 10)$ & $\mathrm{P}>0.5$ \\
\hline Diverting stoma & $70,5 \%(13 / 17)$ & $0 \%(0 / 10)$ & $\mathrm{P}<0,005$ \\
\hline
\end{tabular}

1 colonic perforation was observed in Group B, in 65 years old man, during neoadjuvant therapy for presence of lung and bilobar liver methastasis (Stage IV disease), after 73 days from stent positioning (1/10, equal to $10 \%)$, while in the Group C, one 84 years old man patient, with stage IV disease and frozen pelvis, presented sigmoid perforation after 15 days from stent positioning (1/14, equal to $7,14 \%)$, for this reason this patient was considered in Group C because didin't submitte to operation and died, while the first patient was considered inside the Group B because was submitted to surgery.

Time pocedure was perfomed in 25,17 minutes (range 10-40) and all patients, after 48 hours, were submitted to $\mathrm{Rx}$-Abdomen to control stent position and nobody ( $100 \%$ patients) presented dislocation of SEMS then didn't present bowel obstruction signs.

3 patients of Group B, underwent to neoadjuvant chemotherapy, submitted to radical resection after 94,3 days (range 73-120), while the other 7 patients submitted to radical resection after 7,6 days (range 6-14). No evidence of peritoneal carcinosis and no signs of bowel obstruction didn't show in the Gtoup B. SEMS was perfectly inside the tumor in all patients (Figures 1-4).

T-Student analysis to compare operation time between these two groups (Group A: 192,5 +-72,7 min. vs Group B: 249,12 +- 59,12 min.) shows statistically significance for $\mathrm{T}=2.09$ ( $\mathrm{p}$-value $<0.025$ ).

In terms of survival, from 6 months to 2 years follow-up, we observed, in the control group a survival rate equal to $88 \%(15 / 17)$, in the Group B, $100 \%(10 / 10)$, in the group C, $50 \%$ (7/14) with an average survival of 7,2 months (Range 15 days to 16 months) (Table 3). Even there is a difference beetween Group B vs control group, not statistically significance difference we have found beetween them ( $p$ one tailed 0.42 , two tailed 0.53 , $\mathrm{p}$ value $>0.5$ ), probably due to short time of follow-up.

\section{Discussion}

Since the early 1990s, self- expanding metal stents (SEMS) have been used to treat malignant colonic obstruction. Originally, SEMS was used for palliative purposes as reported by Spinelli et al. describing endoscopic metal stents a simple and effective solution for long-term palliation of rectosigmoid cancer [2].

Recently, it was used as "bridge to surgery" for potentially curable disease, but this kind of procedure is debated in literature. In fact, after initial use of this techniqhe there were some article like that of Fernandez-Esparrach G et al. that reported 22\% of migration of stent, $17 \%$ of obstruction, $7 \%$ perforation and $5 \%$ of tenesmus describing SEMS positioning s procedure to perform only in patients to submitte to surgery for obstructive colorectal cancer [3]. Also D.A.M. Sloothaak et al. in a clinical trials reported $23.07 \%$ of perforation and $30.6 \%$ of adverse effects [4]. On the contrary, during the last few years, many studies showed opposite results as Yang et al. that in a meta-analysis reported the primary anastomosis rate were signifcantly higher in the stent group $(\mathrm{OR}=2.29,95 \% ; \mathrm{p}<0.0001)$, as well as the post-procedural complication rates significantly lower in the stent group $(\mathrm{OR}=0.39,95$, $\mathrm{p}<0.01)$ [5].

Also Nevertheless, Kobborg et al. in 2017 showed a lower complication rate (roughly 20\%) in 239 patients [6].

In our clinical controlled trial, even if is a preliminary data, demonstred that SEMS positioning is a the best palliative procedure with high success rate $(95,8 \%)$ and a good survival without complications for patients affected by obstructive colon cancer, as reported also by Verstockt B et al. describing $94.8 \%$ of success rate 
Giungato S (2019) Perspective Randomized-controlled clinical trial in patients submitted to Self-Expanding Metal Stents for Colo-rectal cancer with bowel obstruction. Single center experience. Preliminary data

Table 3. Comparison between two study groups.

\begin{tabular}{|c|c|c|}
\hline & Group b & Group c \\
\hline N. Patients & 10 & 14 \\
\hline Time procedure min. & $25,17($ range $10-40) \pm 2,22$ & $25,17$ (Range $10-40) \pm 2,22$ \\
\hline Early perforation & $0 \%(0 / 10)$ & $0 \%(0 / 14)$ \\
\hline Long perforation & $10 \%(1 / 10)$ & $1 / 14(7,14 \%)$ \\
\hline Dislocation at 24h & $0 \%$ & $0 \%$ \\
\hline $\begin{array}{c}\text { Operation after sems } \\
\text { (days) }\end{array}$ & $\begin{array}{c}94,3(\text { range } 73-120)^{*} \\
7,6(\text { range } 6-14)^{* *}\end{array}$ \\
\hline
\end{tabular}

*Time elapsed from SEMS positioning to operation after neoadjuvant chemotherapy

"*Time elapsed from SEMS positioning to operation without neodjuvant chemotherapy
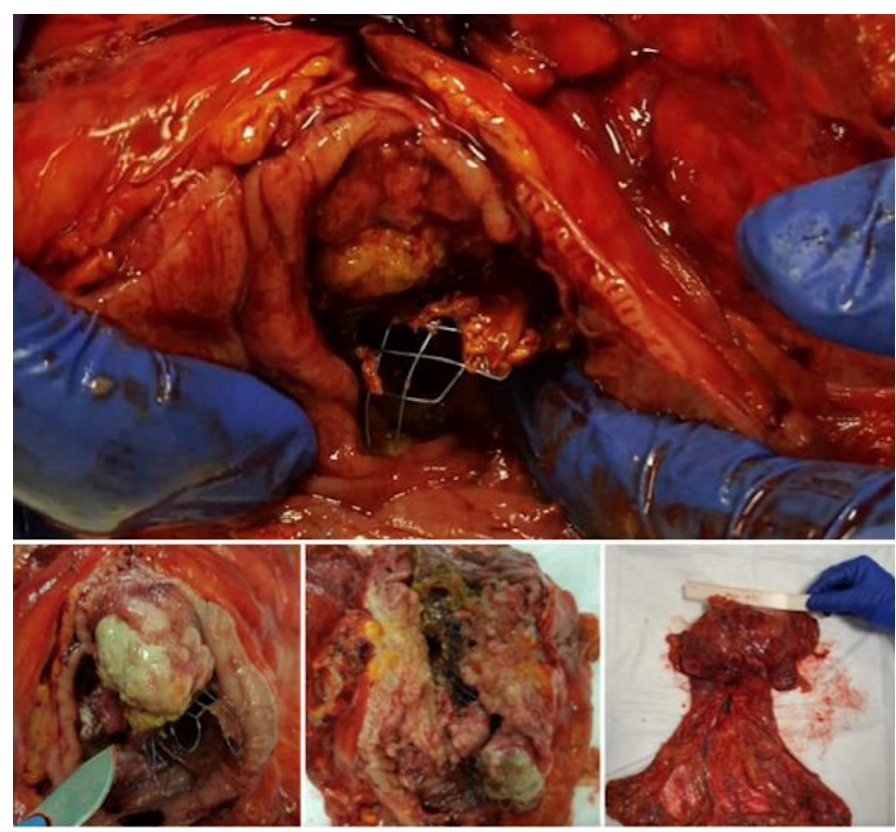

Figure 1. Tranversal resection of patient submitted to self metal stent positioning. Stenti s inside tumor after 5 months

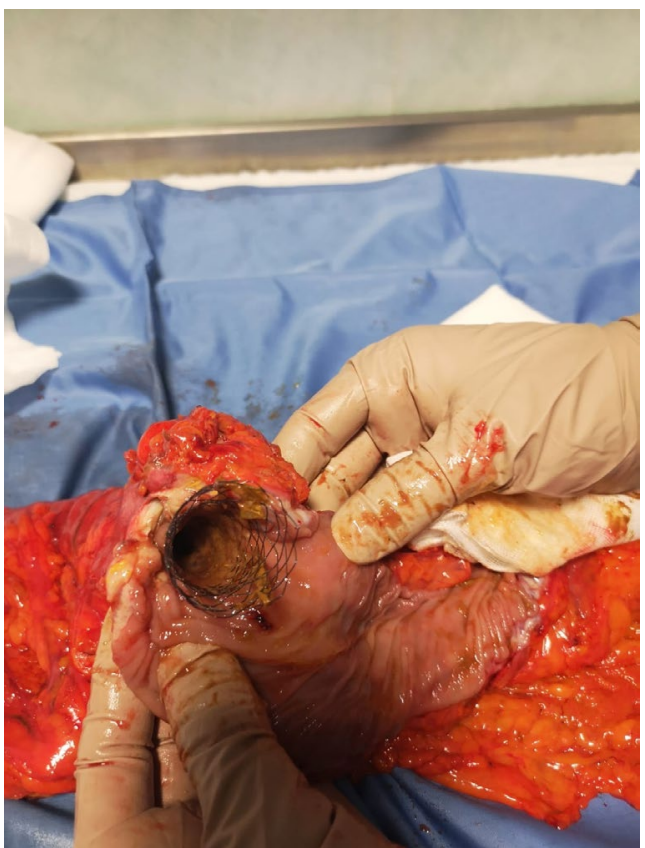

Figure 2. Left hemicolectomy in patient submitted to self expanding metal stent after 7 days

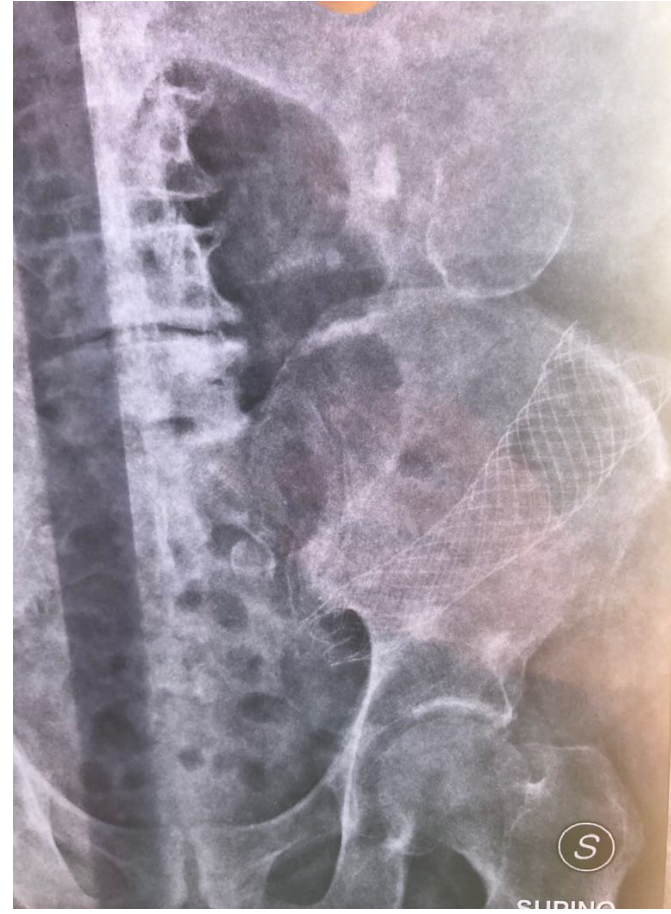

Figure 3. Sigma-decending stent. X-Ray abdomen control after $48 \mathrm{~h}$

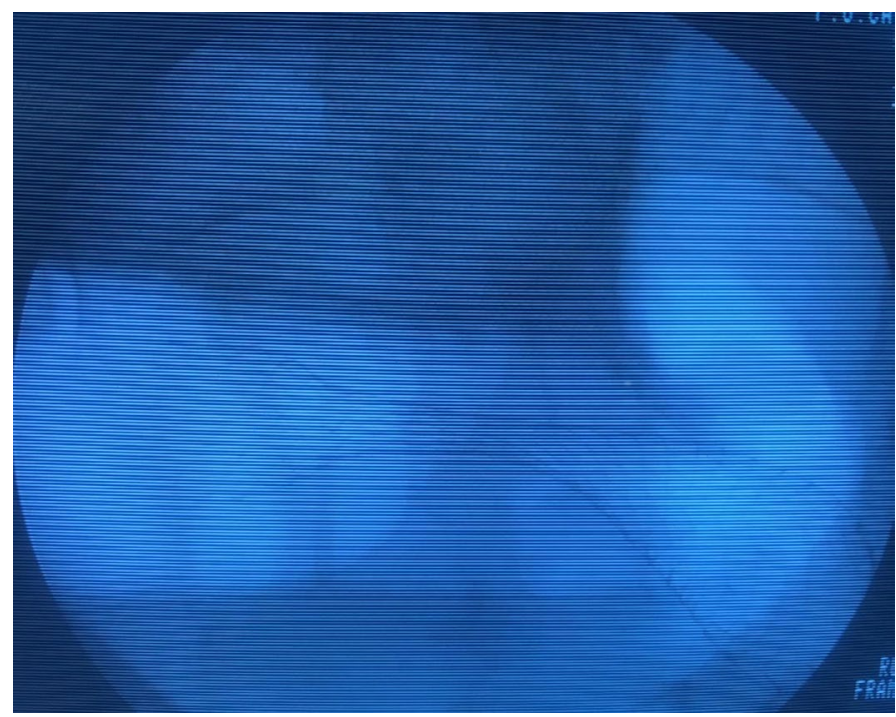

Figure 4. Sigmoid stent. Intraoperative control

with $20 \%$ of complications [7] and by Kobborg $\mathrm{M}$ et al showed only $20 \%$ complication rate [6]. But it is also a valid procedure to perform an elective operation, even laparoscopic, increasing the likelihood of primary anastomosis and reducing the risk of colostomy as reported by Shimizu et al that described mean interval from SEMS insertion to laparoscopic surgery of 21.5 days with no convertion from laparoscopy to open surgery [8] and by Morita $S$ et al. that described a significantly higher rates of primary resection with anastomosis, stoma-free surgery and laparoscopic surgery for patients submitted to SEMS positioning [9].

This randomized study shows the safety and efficacy of colorectal stenting, describing SEMS positioning as the best palliative technique for obstructive colo-rectal cancer with high success rate $(95,8 \%)$ and lower complication rate (17\%) and the analysis of our preliminary data 
Giungato S (2019) Perspective Randomized-controlled clinical trial in patients submitted to Self-Expanding Metal Stents for Colo-rectal cancer with bowel obstruction. Single center experience. Preliminary data

shows a statistical significance $(\mathrm{p}<0.05)$ about the possibility of diverting stoma of patients submitted to emergency surgery and faster operation time $(\mathrm{p}<0.025)$ with feasibility to performe easily laparoscopic resection beacuse the SEMS shows the exact position of tumor.

Furthermore endoscopic metal stent positioning for stage IV disease is a safe and feasibility procedure with short hospitalization, high success rate and survival without complications.

\section{Conclusion}

Our clinical randomized trial shows that SEMS positioning is feasibility as palliative therapy for obstructive colo-rectal cancer and is a safe procedure as "bridge to surgery" because improves both nutrition and physical status providing decompression of the large bowel and functional recovery of bowel. This technique reduces morbidity and mortality for these patients.

Other patients are ruled in this study but they have not been analyzed because are in follow-up or in neoadjuvant chemo therapy, then, a greater follow-up will be necessary to make our study more effective.

\section{Data availability}

The data used to support the findings of this study are available from the corresponding author upon request.

\section{Conflicts of interest}

The authors declare no conflicts of interest.

\section{Authors' contribution}

All authors have contibuted to the design this study. Furthermore, Dr. Fazzolari Laura to draw up discussion and conclusion; Dr. De Luca Giuseppe Massimiliano and Dr. De Luca A. have contributed to literature research; Dr. Palazzo Romana, Dr. Ferrante Michele and Dr. Dimito Camilla have contributed as anesthesiology team; Dr. Asciano Angelo and Dr. Marangolo Francesca have contributed as Radiology team for pre-operative CT-Scan and follow-up X-Ray abdomen after stent positioning; Dr. Brunetti Annalisa have contributed to oncology follow-up of patients; Dr. Pepe AS (head of division) has contributed for controlling the development of study as supervisor.

\section{Acknowledgments}

We thanks all endoscopic and surgical team of "San Pio" Castellaneta Hospital for supporting this study with their work, in particular nursing team of Surgical Unit directed by the head nurse Ms. Borracci D. and Nursing team of Operatory theatre. We also thank endoscopic team composed by Ms. Varegliano L., Ms. Martiello R, Granito $G$ and Boston Scientific team for the technical support received.

Finally we thanks the patients that with their story and their presence for allowing to performe this study.

\section{References}

1. Moher D, Hopewell S, Schultz KF (2010) CONSORTO 2010 explanation and elaboration: updated guidelines for reporting parallel group randomised trials. Int $J$ Surg 10: 28-55.

2. Spineli P, Mancini A (2001) Use of self-expanding metal stents for palliation of rectosigmoid cancer. Gastrointest. Endosc 53: 203-206. [Crossref]

3. Fernandez-Esparrach G, Bordas JM, Giraldez MD, Gines A (2010) Severe complications limit long-term clinical success of self-expanding metal stents in patients with obstructive colorectal cancer. Am J Gastroenterol 105: 1087-1093. [Crossref]

4. DAM Sloothaak, MW van den Berg, MGW Dijkgraaf (2014) Oncological outcome of malignant colonic obstruction in the Dutch stent-in 2 trial. British Jorunal of Surgery 101: 1751-1757. [Crossref]

5. Ping Yang, Xiu-Feng Lin, Wei Li (2018) The role of stents as bridge to surgery for acute left-sided obstructive colorectal cancer: Meta-analysis of randomized controlled trials. Rev Invest Clin 70: 269-278. [Crossref]

6. Kobborg M, Broholm M, Frostberg E, Jeppesen M, Gogenur I (2017) Short-term results of self-expanding metal stents for acute malignant large bowel obstruction. colorectal dis 19: O365-O371. [Crossref]

7. Verstockt B, Van Driessche A, De Man M (2018) Ten-years survival after endoscopic stent placement a a bridge to surgery in obstructing colon cancer. Gastrointest Endosc 87: 705-713.

8. Shimizu H, Yamazaki R, Ohtusuka H (2018) Feasibility of laparoscopic surgery after stent insertion for obstructive colorectal cancer. Asian J Endosc Surg 11: 118-122. [Crossref]

9. Morita S, Yamamoto, Ogawa (2019) Benefits of using a self-expandible metallic stenta s a bridge to surgery for right and left-sided obstructive colorectal cancers. Surg 49: 32-37. [Crossref]

Copyright: $\odot 2019$ Giungato S. This is an open-access article distributed under the terms of the Creative Commons Attribution License, which permits unrestricted use, distribution, and reproduction in any medium, provided the original author and source are credited. 